\title{
Does $360^{\circ}$ lumbar spinal fusion improve long-term clinical outcomes after failure of conservative treatment in patients with functionally disabling single-level degenerative lumbar disc disease? Results of 5 -year follow-up in 75 postoperative patients
}

\author{
Jack E. Zigler, MD ${ }^{\mathrm{a}, *}$, Rick B. Delamarter, MD ${ }^{\mathrm{b}, \mathrm{c}}$ \\ a Texas Back Institute, Plano, TX \\ ${ }^{\mathrm{b}}$ Spine Research Foundation, Santa Monica, CA \\ ${ }^{\mathrm{c}}$ Cedars-Sinai Spine Center, Beverly Hills, CA
}

\begin{abstract}
Background: Surgical treatment of patients with mechanical degenerative disc disease has been controversial, but improvements in clinical outcomes have been shown in properly selected patients with disease-specific diagnoses, with fusion arguably now becoming the "gold standard" for surgical management of these patients. No published study thus far has been designed for prospective enrollment of patients with specific inclusion/exclusion criteria in whom at least 6 months of conservative therapy has failed and who are then offered a standardized surgical procedure and are followed up for 5 years.

Methods: The study group was composed of the patients in the prospective, randomized Food and Drug Administration Investigational Device Exemption trial comparing ProDisc-L (Synthes Spine, West Chester, Pennsylvania) with $360^{\circ}$ fusion for the treatment of single-level symptomatic disc degeneration. Of 80 patients randomized to $360^{\circ}$ fusion after failure of nonoperative care, 75 were treated on protocol with single-level fusions. Follow-up of this treatment cohort was $97 \%$ at 2 years and $75 \%$ at 5 years and serves as the basis for this report. Patients in the trial were required to have failure of at least 6 months of nonoperative care and in fact had failure of an average of 9 months of nonoperative treatment. The mean Oswestry Disability Index score indicated greater than 60\% impairment. The mean entry-level pain score on a visual analog scale was greater than 8 of 10 .

Results: After fusion, not only did patients have significant improvements in measurable clinical outcomes such as the Oswestry Disability Index score and pain score on a visual analog scale but there were also substantial improvements in their functional status and quality of life. Specifically, over $80 \%$ of patients in this study had improvements in recreational status that was maintained 5 years after index surgery, indicating substantial improvements in life quality that were not afforded by months of conservative care. The percentage of patients using narcotics at the 5-year follow-up visit was less than half the percentage of patients who had used narcotics as part of their prior conservative treatment.

Conclusions: The 5-year results of this post hoc analysis of 75 patients involved in a multicenter, multi-surgeon trial support $360^{\circ}$ fusion surgery as a predictable and lasting treatment option to improve pain and function in properly selected patients with mechanical degenerative disc disease. These improvements occurred dramatically immediately after surgery and have been maintained through the scope of this follow-up period, with $98 \%$ follow-up at 2 years and $75 \%$ of patients available at 5 years.
\end{abstract}

(C) 2013 ISASS - International Society for the Advancement of Spine Surgery. Published by Elsevier Inc. All rights reserved.

Keywords: Degenerative disc disease; Lumbar spine; $360^{\circ}$ fusion; 5-Year follow-up

Low-back pain is a fairly ubiquitous condition that remains one of the leading causes for medical treatment in the United States each year. Most acute episodes are due to

\footnotetext{
* Corresponding author: Jack E. Zigler, MD, Texas Back Institute, 6020 W Parker Rd, Ste 200, Plano, TX 75093; Tel: 972-608-5000; Fax: 972608-5020.

E-mail address: jackzigler@juno.com
}

musculoligamentous strains and are self-limited. Even conditions such as herniated discs and flare-ups in patients with underlying degenerative conditions (eg, spondylolisthesis) will frequently respond to rest, medication, and conditioning.

However, there is a small subset of patients who have axial low-back pain due to intrinsic damage to the intervertebral disc. With initial structural damage from mechanical 
overload, biochemical and microstructural changes to water content and proteoglycan content can lead to macrostructural changes such as loss of disc height, annular fissuring, ligamentous laxity, instability, and osteophyte formation. Both chemical and physiological stimuli can trigger secondary muscle spasm and cause inflammatory changes in supporting structures, resulting in pain syndromes that become either constant or increasingly frequent sources of functional disability.

Within the described subset of patients are some who do not improve with standard therapies. They become increasingly disabled and are unable to perform activities that are important to their lifestyles. They also become increasingly reliant on medical therapy (nonsteroidal anti-inflammatory drugs, muscle relaxers, non-narcotic and/or opioid analgesics) and seek progressive levels of conservative care management (chiropractic, physical therapy, pain management injection therapies).

Some of these patients plateau at an unacceptable level, will not or cannot tolerate narcotic analgesic medication as their definitive treatment option, and do not improve. They frequently seek a surgical solution. If single-segment disease can be diagnosed by imaging (radiographic changes of degenerative disc disease [DDD] or instability, magnetic resonance imaging changes in disc quality with or without Modic changes) or by invasive testing (diagnostic blocks, provocative discography), fusion of the affected segment has been the traditional surgical recommendation.

Recent prospective randomized studies of fusion versus nonoperative care have been criticized for various reasons, including their inclusion of patients with a mixed bag of diagnoses, a mixed bag of surgical techniques, and a lack of long-term follow-up. ${ }^{1-4}$ No published study thus far has been designed for prospective enrollment of patients with specific inclusion/exclusion criteria in whom at least 6 months of conservative therapy has failed and who are then offered a standardized surgical procedure and are then followed up scrupulously for 5 years.

Post hoc evaluation of patient outcome data from the ProDisc-L (Synthes Spine, West Chester, Pennsylvania) Food and Drug Administration (FDA) Investigational Device Exemption (IDE) study ${ }^{5}$ offers a unique opportunity to remedy this gap in our literature. Although that study was ostensibly a comparison between ProDisc-L and fusion, it was actually a 3 -armed study. To meet eligibility for inclusion in the study, patients had to have a minimum of 6 months of failed conservative care and an Oswestry Disability Index (ODI) of greater than $40 \%$. The study thus eliminated from consideration the greater pool of patients who improved with conservative care, and it only considered those with failure of a minimum time requirement of treatment while also meeting baseline criteria for impairment as measured by the ODI.

By using 2 of the study arms (failed conservative treatment and $360^{\circ}$ fusion) for a post hoc analysis rather than starting a de novo study, we have essentially been able to make use of the prospectively collected information to jump ahead in time to evaluate these data now. The average patient enrolled in the ProDisc-L versus fusion single-level IDE study had failure of 9 months of conservative care and had an ODI indicating greater than 60\% impairment $(>50 \%$ more impaired for $50 \%$ longer than the minimums required for inclusion). The entry-level pain score on a visual analog scale (VAS) for these patients was greater than 8 of 10 . Of 80 patients randomized to $360^{\circ}$ fusion after failure of nonoperative care, 75 were treated on protocol with single-level fusions. Follow-up of this treatment cohort was $97 \%$ at 2 years and $75 \%$ at 5 years and serves as the basis for this report.

In a prospective study of a large group of patients receiving conservative care, such care would be expected to relieve symptoms in a majority of those enrolled. We acknowledge this, and the percent responding to conservative care, who were never considered for inclusion in this study, is not the issue of this report. Rather, this analysis begins its evaluation at the next branch point in the algorithm, asking the following question: Among those in whom nonoperative treatment fails, is surgical treatment a better option than accepting their level of disability on a continuing conservative care management program? We believe that this study provides the data to answer this question. We can show the immediate postoperative improvement compared with the baseline of 9 months of failed conservative care and can document maintenance of those clinical outcome improvements out to 5 years.

\section{Methods}

Patients with symptomatic single-level DDD were treated in this randomized, controlled, multicenter FDA clinical trial that evaluated total disc replacement (TDR) (ProDisc-L) compared with circumferential fusion. The major inclusion criteria were as follows: skeletally mature individuals with functionally disabling radiographically proven DDD at 1 vertebral level between L3 and S1 (by plain radiographs, magnetic resonance imaging scan, computed tomography scan, or discography), in whom conservative treatment for a minimum of 6 months had failed, who had back and/or leg (radicular) pain, and who had a minimum ODI score of $40 \%$ impairment or greater. The main exclusion criteria were as follows: patients with greater than grade I spondylolisthesis, previous lumbar fusion, $\mathrm{T}$ score on dual-energy $\mathrm{x}$-ray absorptiometry scan worse than -1.0 , or clinically relevant facet joint degenerative disease.

Seventeen sites participated in the study. The average patient enrolled in this study had a VAS pain score greater than 8 of 10, had an ODI of 63\%, and was symptomatic for 9 months. Patients at each site were randomized in a 2:1 ratio of TDR to circumferential fusion. Separate randomization schedules were generated for each of the 17 sites using a fixed block size of 6 . The randomization was held by the sponsor and disclosed to the site only after individual 
Table 1

Patient treatment allocation and follow-up

\begin{tabular}{ll}
\hline & Description \\
\hline Allocation & 93 were enrolled \\
& 75 received intervention \\
& 18 did not receive intervention \\
& - 2 denied insurance coverage \\
& - 8 changed their minds \\
& - 3 were no longer surgical candidates at time of surgery \\
& • 5 were treated off protocol \\
& 73 were evaluated (97.1\% follow-up) \\
& - 2 secondary surgical interventions (included in primary \\
& composite endpoint, excluded from secondary analyses) \\
& 2 missed visits (excluded from analysis) \\
& 56 were evaluated (74.7\% follow-up) \\
& • 5 secondary surgical interventions (included in primary \\
& composite endpoint, excluded from secondary analyses) \\
& 19 missed visits (excluded from analysis) \\
& - 1 death \\
& $\bullet 18$ were lost to follow-up
\end{tabular}

patient enrollment, including signed written informed consent provided by the patient. Patients were blinded to randomization until immediately after surgery.

A comparison of the outcomes of patients prospectively randomized to ProDisc-L versus fusion has been reported at 2 years' follow-up ${ }^{5}$ and at 5 years' follow-up, ${ }^{6,7}$ but this is the first report evaluating outcomes of the $360^{\circ}$ fusion group compared with their own failed-conservative therapy baselines.

A total of 80 of the patients in whom nonoperative treatment failed were randomized to fusion and treated surgically. Five patients were treated off protocol because of intraoperative decision making by their surgeons, who determined during surgery that a 2-level fusion was more appropriately indicated and was therefore performed. These patients treated off protocol were not included in efficacy data per FDA guidelines and are not considered in this study. The 75 patients treated on protocol who were followed up for 5 years are reported in this post hoc analysis.

The fusion group received commercially available femoral ring allograft with or without demineralized bone matrix as their interbody construct, as well as posterolateral fusion with autogenous iliac crest bone graft in combination with pedicle screws. No co-interventions were used. Per IDE protocol, patients were evaluated preoperatively and postoperatively at 6 weeks and 3, 6, 12, 18, and 24 months. After completing 24 months of follow-up, patients were asked to consent (with appropriate institutional review board approval) to additional follow-up visits at 36,48 , and 60 months.

The same evaluative protocol was followed in all patients at all sites at each visit, with physical and neurologic examinations, radiographs, and patient self assessments including the ODI, ${ }^{8}$ Short Form Health Survey (Short Form 36 [SF-36]), pain intensity on a 100-mm VAS (VAS pain), VAS for patient satisfaction, and whether the patient would have the same surgery again. A flowchart of patient allocation and follow-up is shown in Table 1.

Intraoperative data were previously reported in detail. ${ }^{9}$ The mean intraoperative time in the fusion group was 229.0 minutes. The mean estimated blood loss was $466.8 \mathrm{~mL}$. The length of hospital stay in the fusion group across all 17 centers was 4.3 days. Demographics of the treatment group are shown in Table 2.

\section{Results}

\section{Primary clinical outcomes}

\section{Oswestry Disability Index}

Preoperative ODI scores averaged $63 \%$ in the patients in whom conservative treatment failed and who went on to have fusion (Table 3). At their earliest postoperative visits, these patients showed an immediate and significant improvement compared with their baseline ODI scores, and this improvement persisted out to 5 years, at all times remaining statistically significantly better than their status after 9 months of conservative treatment. At 2 years, $64.8 \%$ of fusion patients had $15 \%$ or greater improvement in the ODI score and $54.9 \%$ had a 15 -point or greater improvement in ODI score.

At 5 years, the fusion group maintained statistically significant improvements in ODI scores compared with baseline $(P<.0001)$. The mean ODI score improvements for fusion patients were maintained from 2 to 5 years. The

Table 2

Demographics and baseline characteristics of all patients enrolled and treated on protocol

\begin{tabular}{lc}
\hline & Data $(\mathrm{n}=75)$ \\
\hline Age at surgery [mean (SD)] (y) & $40.4(7.6)$ \\
Male [n (\%)] & $34(45.3 \%)$ \\
Race [n (\%)] & $59(78.7 \%)$ \\
$\quad$ White & $5(6.7 \%)$ \\
African American & $10(13.3 \%)$ \\
Hispanic & $0(0.0 \%)$ \\
Asian American & $1(1.3 \%)$ \\
Other & \\
Smoking status & $34(45.3 \%)$ \\
Never & $17(22.7 \%)$ \\
Former & $24(32.0 \%)$ \\
Current & $27.3(4.4)$ \\
Body mass index [mean (SD)] $\left(\mathrm{kg} / \mathrm{m}^{2}\right)$ & $62.7(10.3)$ \\
ODI score [mean (SD)] (out of 100$)$ & $57(76.0 \%)$ \\
Narcotic use as prior treatment [n $(\%)]$ & $37(49.3 \%)$ \\
Engaged in recreational activity [n $(\%)]$ & \\
Implant level [n (\%)] & $3(4.0 \%)$ \\
L3-L4 & $22(29.3 \%)$ \\
L4-L5 & $50(66.7 \%)$ \\
L5-S1 & $23(30.7 \%)$ \\
Prior surgical treatment [n (\%)] & \\
\hline
\end{tabular}

NOTE. Continuous and ordinal variables were analyzed by a Wilcoxon rank sum test; categorical variables were analyzed with the Fisher exact test to compare Fusion single level Randomized patients (F1R) with ProDisc-L single level Randomized patients (P1R). 
Table 3

ODI scores at baseline, 2 years, and 5 years

\begin{tabular}{llll}
\hline & \multicolumn{2}{l}{ ODI score } & \\
\cline { 2 - 4 } & $\mathrm{n}$ & Mean (SD) & \% Change (SD) \\
\hline Baseline & 75 & $62.7(10.3)$ & - \\
$2 \mathrm{y}$ & 71 & $39.8(24.3)$ & $-37.8 \%(36.0 \%)$ \\
$5 \mathrm{y}$ & 51 & $36.2(25.7)$ & $-43.8 \%(37.1 \%)$
\end{tabular}

NOTE. The Wilcoxon test was used to compare baseline data; $t$ tests were used to compare 24- and 60-month percent improvement.

majority had ODI score improvements of $15 \%$ or greater at 5 years $(76.0 \%$ of fusion patients) or improvements of 15 points or greater at 5 years $(64.0 \%$ of fusion patients). Of the fusion patients who had ODI score improvements of $15 \%$ or greater at 2 years compared with baseline, $83.3 \%$ maintained a $15 \%$ or greater improvement from baseline at 5 years.

\section{SF-36 physical component summary}

The mean baseline SF-36 physical component summary (PCS) score and improvements at 2 and 5 years in fusion patients are shown in Table 4 . At 2 years, $70.0 \%$ of fusion patients had maintenance or improvement in SF-36 PCS scores. At 5 years, the majority of fusion patients $(72.6 \%)$ had maintenance or improvement in SF-36 PCS scores compared with baseline. Of the patients with maintained or improved SF-36 PCS scores compared with baseline at 2 years, $83.3 \%$ also had maintenance or improvement out to 5 years.

\section{Neurologic success}

Neurologic success was defined as the maintenance or improvement of patient responses to all neurologic criteria: sensory, motor status, reflexes, and a straight-leg raise test. At 2 years, the fusion group showed success in $81.4 \%$ (57 of 70 patients). Compared with 2 years, the percentage of patients achieving overall neurologic success at 5 years increased in fusion patients (43 of 48 patients, 89.6\%). Of the 13 fusion patients who were considered to have neurologic failure at 2 years, 6 were found to have neurologic success at 5 years.

\section{Radiographic outcomes}

Five radiographic outcome components, determined by independent radiologists based on digitized films, refer to

Table 4

SF-36 PCS scores

\begin{tabular}{llll}
\hline & \multicolumn{2}{l}{ SF-36 PCS score } & \\
\cline { 2 - 4 } & $\mathrm{n}$ & Mean (SD) & \% Change (SD) \\
\hline Baseline & 74 & $30.9(5.6)$ & - \\
$2 \mathrm{y}$ & 70 & $38.8(11.3)$ & $29.8 \%(40.9 \%)$ \\
$5 \mathrm{y}$ & 51 & $40.1(13.6)$ & $29.9 \%(43.7 \%)$
\end{tabular}

NOTE. The Wilcoxon test was used to compare baseline data; $t$ tests were used to compare 2- and 5-year percent improvement.
Table 5

Radiographic outcomes at 5 years

\begin{tabular}{ll}
\hline & No. of patients \\
\hline No device migration & $45 / 45(100.0 \%)$ \\
No device subsidence & $45 / 45(100.0 \%)$ \\
Disc height decrease $\leq 3 \mathrm{~mm}$ & $41 / 45(91.1 \%)$ \\
Fusion status & $43 / 45(95.6 \%)$ \\
No radiolucency & $45 / 45(100.0 \%)$ \\
Range of motion & $44 / 44 *(100.0 \%)$ \\
\hline
\end{tabular}

* One fusion patient was missing extension radiographs.

qualitative evaluations of device migration, device subsidence, disc height maintenance, fusion status, and radiolucency (Table 5). The results presented in this section describe those for patients with available radiographs (45 of 75) for the 5-year follow-up visit.

At 5 years, none of the fusion patients with available films had observed anterior construct migration. There were 4 fusion patients with observed disc height decrease ( $>3$-mm decrease in disc height due to subsidence or graft settling) at the 5-year visit. There were no cases of subsidence (ie, violation of the vertebral endplates $>3 \mathrm{~mm}$ ) or radiolucency observed in any of these fusion patients at 5 years.

Radiographic films available at 5 years indicated that 2 fusion patients had failure to achieve greater than $50 \%$ trabecular bridging bone, although neither of these patients required a secondary procedure for clinical pseudarthrosis. In other words, although 2 patients did not meet the very strict radiographic criteria for success imposed by the study parameters, neither displayed clinical evidence of nonunion and neither required additional intervention. Both were considered clinically fused by their surgeons.

The radiographic fusion rates were $97.1 \%$ and $95.6 \%$ of fusion patients with available films at 2 and 5 years, respectively. All fusion patients had less than $3 \mathrm{~mm}$ of translation and less than $5^{\circ}$ of flexion/extension rotation observed on 5-year radiographs.

Index-level secondary surgeries

Secondary surgeries at the index level occurred in 9 fusion patients $(12 \%)$ by the end of the 5-year study. Details regarding these patients will be described in this section.

Device success was defined as absence of any reoperation required to modify or remove implants and no need for supplemental fixation. At 2 years, device success was achieved in $97.3 \%$ of the fusion patients. At 5 years, device success was achieved in $93.3 \%$ of the fusion patients.

The 2 fusion patients who were considered to have device failure at 2 years both had unresolved pain requiring posterior reoperation (2.7\%). Between years 2 and 5, 3 additional patients were considered to have device failure because they also had unresolved pain that required reoperation (6.7\% cumulative).

Four additional fusion patients underwent secondary surgeries at the index level by 5 years of follow-up but were 
Table 6

Patient pain assessment using 100-mm VAS

\begin{tabular}{llll}
\hline \multicolumn{4}{l}{ VAS pain score } \\
\cline { 2 - 4 } & $\mathrm{n}$ & Mean (SD) & \% Change (SD) \\
\hline Baseline & 73 & $74.9(14.7)$ & - \\
$2 \mathrm{y}$ & 71 & $43.3(31.6)$ & $-42.4 \%(42.9 \%)$ \\
$5 \mathrm{y}$ & 51 & $40.0(32.1)$ & $-47.5 \%(43.8 \%)$ \\
\hline
\end{tabular}

NOTE. The Wilcoxon test was used to compare baseline data; $t$ tests were used to compare 24- and 60-month percent improvement.

not considered to have device failure because the device or intended treatment was not altered in any way. These patients underwent routine hardware removal for pain. The fusion mass in these patients was confirmed to be solid during the surgery.

Of the 9 of 75 patients requiring index-level reoperations during this 5-year window, 6 underwent reoperations for simple hardware removal because of local posterior implant-related pain. Their fusions were solid, and no additional interventions were required during the scope of their observation windows. In clinical practice, hardware removal is not an uncommon or particularly dangerous procedure after healing of a pedicle screw-augmented posterolateral fusion.

\section{Secondary clinical outcomes}

\section{VAS pain}

A summary of VAS pain assessment is provided in Table 6. Fusion patients showed statistically significant improvements in VAS pain scores at both 2 years and 5 years compared with baseline $(P<.0001)$. The mean percent improvements in VAS pain scores were similar at the 2-year and 5-year follow-up visits.

\section{Patient satisfaction}

Patients indicated their satisfaction with treatment on a 100-mm VAS. At 2 years, the mean VAS satisfaction score in fusion patients measured $67.3 \pm 31.5$. At 5 years, the mean VAS satisfaction score increased to $77.5 \pm 26.8$. When asked whether they would have the same surgery again, $68.0 \%$ of fusion patients responded yes at 5 years.

\section{Recreational activity status}

At baseline, only $49.3 \%$ of 75 fusion patients reported that they engaged in recreational activities. At 2 years, the recreational status improved, with $78.3 \%$ of 69 fusion patients now able to enjoy their recreational activities. At 5 years, $90.0 \%$ of 50 fusion patients were able to return to recreation. This represents tremendous improvement in patients who were severely functionally impaired despite 9 months of conservative care management. From a clinician caregiver's perspective, this represents a very strong argument for success after surgical intervention with fusion in these patients.

\section{Narcotic use}

At the time of surgery, $76 \%$ of fusion patients had used narcotics as a form of prior conservative treatment. The percentage of patients taking narcotics decreased from baseline at 2 years (42.5\% of 70 fusion patients) and remained diminished at 5 years $(40.0 \%$ of 50 fusion patients). This nearly $50 \%$ reduction in narcotic use in a patient population who would otherwise have required lifetime narcotic use is also a strong indicator that fusion offers a better outcome. Pharmaceutical cost savings even within this 5-year window, let alone the projected lifetime savings, are substantial.

\section{Complications}

At the completion of the study, severe or life-threatening adverse events were reported at 0.39 per patient.

Two fusion group patients had clinically significant blood loss $(>1500 \mathrm{~mL})$ due to iliac vein tears during the index surgery. Both were addressed successfully at the time of index surgery with no adverse clinical sequelae. In addition, 2 fusion patients had intraoperative dural tears, both of which were treated successfully at index surgery without clinical sequelae. Postoperatively, retrograde ejaculation was reported in 1 patient. Two posterior wound infections required treatment in the group. Deep venous thrombosis developed after surgery in 1 patient and was successfully treated medically. At 5 years, 1 fusion patient had died for reasons unrelated to the surgery.

\section{Discussion}

This post hoc analysis of the failed-conservative care patients who were randomized to the fusion control group of the ProDisc-L versus $360^{\circ}$ fusion FDA IDE study builds upon the reported 2-year results of that study and continues follow-up of the control patient cohort through its 5-year postmarket surveillance. Comparison of the investigational and control surgical cohorts supported the primary hypothesis of this multicenter, prospective, randomized trial at both 2 years and 5 years, allowing the conclusion that TDR is not inferior to fusion.

This analysis of the clinical outcomes of the control patients compared with their own baseline conservative care data clearly shows the benefits of fusion in appropriately selected patients in whom conservative care has failed. Seventy-five fusion patients in whom an average of 9 months of prior nonoperative care had failed showed improved postoperative clinical outcomes over baseline and were followed up at 2 years and 5 years. Fusion patients had further improvement in mean clinical outcomes from 2 to 5 years, with mean outcomes reaching levels similar to those observed for TDR patients at 5 years.

In this study a successful fusion status required strong radiographic evidence of fusion, showing more than $50 \%$ trabecular bridging bone or bone mass maturation, increased or maintained bone density at the site, and no visible gaps in the fusion mass. Using these strict radiographic 
criteria, independent radiologists determined that the fusion rate in these patients was $97.1 \%$ at 2 years and $95.6 \%$ at 5 years. Our fusion rates compare quite favorably with those reported previously for instrumented fusion in patients undergoing treatment for DDD in other prospective, randomized studies $(73 \%-95 \%){ }^{9-12}$

It is noteworthy that the patients in this study received femoral ring allograft and autogenous iliac crest bone graft but no bone morphogenetic protein (BMP) (BMP was not an FDA-approved product at the inception of this study), and fusion rates in this series of patients were higher than those reported in prospective, randomized studies evaluating BMP in single-level DDD patients (88\% reported by Dimar et $\mathrm{al}^{11} ; 91 \%$ reported by Burkus et $\mathrm{al}^{13}$ ). These findings further support the quality of treatment rendered to our fusion group.

Patient selection followed clear inclusion and exclusion criteria. More than 30 surgeons at 17 different sites participated in this study, so these results should be generalizable to community spine practices across the United States. Appropriate patient selection and technically well-performed anterior-posterior fusion, well within the scope of practice of dedicated spine surgeons, should yield similar clinical outcomes.

Contrary to increasingly strong outcome-based evidence in the scientific literature reporting benefits of fusion, recent editorials in the medical literature and lay-media commentaries continue to denounce surgery as a viable option for disabling DDD. One unfortunate consequence is that surgical authorization policies by national insurance payers are becoming narrower, limiting coverage for reconstructive spine surgery in patients who are having persisting functional disability as a result of mechanical DDD. Our results, combined with other strong scientific evidence in the more recent peer-reviewed scientific literature, strongly indicate a need for reconsideration of this anecdotal conservative posture. In this study highly disabled patients with mechanical DDD in whom conservative treatment for at least 6 months failed were treated very successfully with spinal fusion. Their improvements immediately after surgery remained stable, and in some instances continued to improve, over the 5 -year period of observation that we describe. This was the experience of over 30 surgeons at 17 different centers across the United States.

After fusion, not only did patients have significant improvements in measurable clinical outcomes such as the ODI score and VAS pain score but there were also substantial improvements in their functional status and quality of life. Specifically, over $80 \%$ of patients in this study had improvements in recreational status that was maintained 5 years after index surgery, indicating substantial improvements in life quality that were not afforded by months of conservative care. The percentage of patients using narcotics at the 5-year follow-up visit was less than half the percentage of patients who had used narcotics as part of their prior conservative treatment.
Data from this study clearly show that in an appropriately selected patient (with adherence to strict inclusion/ exclusion criteria), spinal surgery is indeed a very good treatment option for improving multiple parameters for life quality (such as function, disability, and pain). In this patient group, significant improvements over lifetime conservative care can be shown, with concomitant benefits to society in terms of a more productive population, decreased reliance on narcotic analgesic medication, and a reduction in lifetime medical costs.

\section{Conclusions}

The 5-year results of this post hoc analysis of 75 patients involved in a multicenter, multi-surgeon trial support $360^{\circ}$ fusion surgery as a predictable and lasting treatment option to improve pain and function in properly selected patients with mechanical DDD. These improvements occurred dramatically immediately after surgery and have been maintained through the scope of this follow-up period, with $98 \%$ follow-up at 2 years and $75 \%$ of patients available at 5 years. Proper patient selection requires strict adherence to inclusion/exclusion criteria, a firm diagnosis of anterior column discogenic pain origin, and a failure of at least 6 months of conservative therapy. Fusion offers a much better treatment alternative than failed conservative care, with benefits that are measurable, immediate, and long-lasting.

\section{References}

1. Fritzell P, Hägg O, Wessberg P, Nordwall A, Swedish Lumbar Spine Study Group. Chronic low back pain and fusion: a comparison of three surgical techniques: a prospective multicenter randomized study from the Swedish lumbar spine study group. Spine (Phila Pa 1976) 2002; 27:1131-41.

2. Fritzell P, Hägg O, Nordwall A, Swedish Lumbar Spine Study Group. Complications in lumbar fusion surgery for chronic low back pain: comparison of three surgical techniques used in a prospective randomized study. A report from the Swedish lumbar spine study group. Eur Spine J 2003;12:178-89.

3. Weinstein JN, Lurie JD, Tosteson TD, et al. Surgical versus nonsurgical treatment for lumbar degenerative spondylolisthesis. $\mathrm{N} \mathrm{Engl}$ J Med 2007;356:2257-70.

4. Weinstein JN, Lurie JD, Tosteson TD, et al. Surgical compared with nonoperative treatment for lumbar degenerative spondylolisthesis. Fouryear results in the spine patient outcomes research trial (SPORT) randomized and observational cohorts. J Bone Joint Surg Am 2009;91:1295-304.

5. Zigler J, Delamarter R, Spivak JM, et al. Results of the prospective, randomized, multicenter Food and Drug Administration investigational device exemption study of the ProDisc-L total disc replacement versus circumferential fusion for the treatment of 1-level degenerative disc disease. Spine (Phila Pa 1976) 2007;32:1155-62.

6. Zigler JE, Delamarter RB. Five-year results of the prospective, randomized, multicenter, Food and Drug Administration investigational device exemption study of the ProDisc-L total disc replacement versus circumferential arthrodesis for the treatment of single-level degenerative disc disease. J Neurosurg Spine 2012;17:493-501.

7. Zigler JE. Five-year results of the ProDisc-L multicenter, prospective, randomized, controlled trial comparing ProDisc-L with circumferential spinal fusion for single-level disabling degenerative disk disease. Semin Spine Surg 2012;24:25-31. 
8. Hudson-Cook N, Tomes-Nicholson K, Breen A. A revised Oswestry disability questionnaire. In: Back pain: new approaches to rehabilitation and education. Manchester: Manchester University Press; 1989: 187-204.

9. Fischgrund JS, Mackay M, Herkowitz HN, Brower R, Montgomery DM, Kurz LT. 1997 Volvo Award winner in clinical studies. Degenerative lumbar spondylolisthesis with spinal stenosis: a prospective, randomized study comparing decompressive laminectomy and arthrodesis with and without spinal instrumentation. Spine (Phila Pa 1976) 1997;22:2807-12.

10. Zdeblick TA. A prospective, randomized study of lumbar fusion. Preliminary results. Spine (Phila Pa 1976) 1993;18:983-91.
11. Dimar JR, Glassman SD, Burkus KJ, Carreon LY. Clinical outcomes and fusion success at 2 years of single-level instrumented posterolateral fusions with recombinant human bone morphogenetic protein-2/ compression resistant matrix versus iliac crest bone graft. Spine (Phila Pa 1976) 2006;31:2534-9.

12. Chung SK, Lee SH, Lim SR, et al. Comparative study of laparoscopic L5-S1 fusion versus open mini-ALIF, with a minimum 2-year followup. Eur Spine J 2003;12:613-7.

13. Burkus JK, Gornet MF, Schuler TC, Kleeman TJ, Zdeblick TA. Six-year outcomes of anterior lumbar interbody arthrodesis with use of interbody fusion cages and recombinant human bone morphogenetic protein-2. J Bone Joint Surg Am 2009;91:1181-9. 